\title{
XIV.
}

\section{Die Aktivbedeutung der Adjektiva auf bilis im archaischen Latein.}

Der Gegenstand, den ich behandeln will, ist ein sehr specieller, aber ich glaube, daß durch seine Aufhellung sich Gesichtspunkte ergeben werden, welche nicht nur für dies beschränkte, sondern auch für weitere Gebiete der lateinischen Grammatik fruchtbar werden können.

Wie andern Kapiteln der lateinischen Wortbildungslehre, so hat auch den Adjektiven auf -bilis Paucker seine Sorgfalt zugewendet. In seinem Aufsatz über "die Verbaladjektiva auf -bilis" in den "Vorarbeiten zur lateinischen Sprachgeschichte " Berlin 1884 S. 46 ff. gibt er ein alphabetisches Verzeichniß sämmtlicher Adjektiva auf -bilis mit kurzem Vor - und Nachwort. Doch gewährt diese Arbeit, obwohl durch den Druck kenntlich ist, welchem Zeitalter die Adjektiva angehören und ob sie aktive oder passive oder beide Bedeutungen haben, für meine

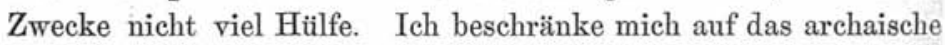
Latein und gebe ein ungefähr bis zum Jahre 100 vor Chr. reichendes Verzeichniß der Stellen, wo Adjektiva auf -bilis vorkommen.

\section{Adjectiva auf bilis mit echter Passivbedeutung.}

Als echtes Passiv bezeichne ich das gewöhnliche aus den Passivformen des Verbums genugsam bekannte im Unterschied 
von gewissen Mittelstufen zwischen Activ und Passiv, welche ich weiterhin $\mathrm{zu}$ besprechen haben werde. Zur Passivität gesellt sich in den Adjektiven auf bilis die Modalbedeutung der Möglichkeit. Aber letztere weist zwei Nuancen auf: merx invendibilis (Plaut. Poen. 341) und homo infortunio donabilis (Plaut. Rud. 654) stehen nicht auf einer Stufe, ersteres heißt „eine Waare, die nicht verkauft werden kann", letzteres ,ein Mensch, der werth ist mit Unglück beschenkt zu werden". Man ist oft versucht, in diese zweite Species die Modalbedeutung der Nothwendigkeit hineinzutragen, aber das wäre nicht richtig: homo infortunis donabilis ist nicht, ein Mensch, der mit Unglïck beschenkt werden $m u \beta$ " sondern ,ein Mensch, der mit Unglüick beschenkt werden kann, weil er dessen werth ist". Ich will, um diese beiden Arten der Modalität zu scheiden, die Ausdrücke wirkliche Möglichkeit und moralische Möglichkeit anwenden ${ }^{1}$ ) und nehme die Beispiele, welche die wirkliche Möglichkeit zeigen, voraus ${ }^{2}$ ) : Terenz Adelph. 896 Meditor esse adfabilis. Plaut. Stich. 74 Exorabilist (scil. pater, vielleicht exorabilest zu schreiben) Plaut. Cas. 4, 3, 18 Edepol nae tu, si esses equus, esses indomabilis. Accius tr. 75 Quid hic tam obscure dictum est tamve inenodabile. Pacuv. tr. $122 \mathrm{Ni}$ me inexorabilem faxis. Terenz Phorm. 497 Adeon ingenio esse duro te atque inexorabili. Plaut. Aul. 191 Virginem habeo grandem, dote cassam atque inlocabilem. Accius tr. 91 Pertolerarem vitam cladesque exanclarem inpetibilis. XII tab. 8, 22 (Schöll) Qui se sierit testarier libripensve fuerit, ni testimonium fatiatur, inprobus intestabilisque esto (testabilis wird aus einer lex Horatia citirt von Gellius 7, 7, 2. 3). Plaut. Curc. 30 Semper curato ne sis intestabilis. Plaut. Mil. 1414 Et si hinc non abeo intestatus, bene agitur pro noxia. - Quid, si id non faxis? - Ut vivam semper intestabilis. Plaut. Aul. 533 Haec

1) Der Begriff der Nothwendigkeit kann in zwei entsprechende Unterabtheilungen zerfallen; dieselben kommen z. B. zum Ausdruck in der Verschiedenheit, welche zwischen necesse est und oportet besteht.

2) Die Adjektive sind alphabetisch geordnet. Ich citire Plautus Menaechmi, Mostellaria, Persa nach Ritschl, Casina und Cistellaria nach Weise, die übrigen Stücke nach den von Götz, Schöll, Löwe besorgten Ausgaben (doch habe ich von Pseudolus die Ritschl'sche, von Captivi die Sonnenschein'sche, von Rudens die Fleckeisen'sche Ausgabe benutat und nur nachträglich meine Citate den neuen inzwischen erschienenen; Ausgaben angepaßt), Terenz nach Umpfenbach, die Tragiker und Komiker nach Ribbeck, die übrigen Dichter nach Bährens, Cato de re rust. nach Keil, Cato's Fragmente nach Jordan. 
sunt atque aliae multae in magnis dotibus incommoditates sumptusque intolerabiles. Afran. tog. 255 Didicisset ferre et non esse intolerabilis. Plaut. Poen. 341 Invendibili merci oportet ultro emptorem adducere. Ennius com. 1 Huic est animus propitiabilis. Plaut. Cist. 1, 1, 63 Facito ut facias stultitiam sepelibilem (bringe deiné Thorheit dahin, daß sie begraben werden kann). Plaut. Capt. 518 Hic illest dies, quom nulla vitae meae salus sperabilist. Terenz heaut. tim. 205. Paulo qui est homo tolerabilis.

Dagegen erkenne ich die moralische Möglichkeit in folgenden Beispielen: Plaut. Capt. 302 Vis hostilis cum istoc fecit meas opes aequabiles (aequabilis heißt hier wohl noch , werth gleichgestellt zu werden", in classischer Zeit ist es in die intransitive Bedeutung ,gleich" ïbergegangen). Plaut. Curc. 168 Quid vidisti aut quid videbis magis dis aequiperabile. Plaut. Trin. 466 Ita nunc tu dicis, non esse aequiperabilis vostras cum nostris factiones atque opes? Plaut. Asin. 674 Nimis bella's atque amabilis. Plaut. Stich. 737 Mea suavis, amabilis, amoena. Accius tr. 555 Aspernabilem ne haec taetritudo mea me inculta faxit. Plaut. Trin. 44 Qui admisit in se culpam castigabilem. Plaut. Pseud. 525 Dabo aliam pugnam claram et commemorabilem. Plaut. Bacch. 616 Credibile hoc est? (Worte wie credibilis, bei welchem die wirkliche und die moralische Möglichkeit fast immer zusammenfällt, haben wohl den AnstoB zur Ausbildung des Begriffes der moralischen Möglichkeit gegeben). Plaut. Trin. 606 Non credibile dicis. Terent. Andr. 625 Hocinest credibile aut memorabile? Plaut. Rud. 654 Edepol infortunio hominem praedicus donabilem. Plaut. Cist. 4, 1, 1 Nullam ego me vidisse credo magis anum excruciabilem. Accius Tr. 270 Tyranni saevom ingenium atque execrabile. Plaut. Stich. 395 Ego huc citus praecucurri, ut nuntiarem nuntium exoptabilem (eine Nachricht, welche werth ist erwünscht zu sein). Lucilius 467 (Bährens) Neque inimicis invidiosam neque amico exoptabilem. Plaut. Bacch. 614 Inamabilis, inlepidus vivo. Plaut. Rud. 912 Miroque modo atque incredibili hic piscatus mihi lepide evenit (vielleicht wäre es richtiger, hier wirkliche Möglichkeit anzunehmen). Terenz Eun. 1049 Incredibilia Parmeno modo quae narravit! Terenz Heaut. Tim. 624 Vin me istuc tibi, etsi incredibilest, credere?. Terenz Phorm. 239 Quia praeter spem atque incredibile hoc mihi obtigit. Terenz Phorm. 247 Incredibilest quantum erum ante eo sapientia. Terenz Hec. 377 
Incredibili re atque atroci percitus. Com. inc. 40 Incredibile hoc factum obicitur. Plaut. Bacch. 614 Incredibilis imposque animi inamabilis inlepidus vivo (incredibilis ïbersetzt Pareus im Lexicon Plautinum ,indignus cui quisquam credat", es ist ein Beispiel für eine Passivbildung, bei welcher das dativische Objekt des Aktivs zum Subjekt des Passivs erhoben wird wie sonst das akkusativische). Auct. prol. Plaut. Capt. 56 Neque spurcidici insunt versus inmemorabiles. Plaut. Capt. 684 At erit mi hoc factum mortuo memorabile. Plaut. Curc. 8 Istuc quidem nec bellumst nec memorabile. Plaut. Stich. 729 Hoc memorabilest: ego tu sum, tu's ego. Terenz Andr. 625 Hocinest credibile aut memorabile? Terenz Heaut. tim. 314 Non fit. sine periclo facinus magnum nec memorabile. Accius tr. 613 Virum memorabilem intui viderer (venerabilem vermuthet Bücheler). Plaut. Epid. 225 Quid istuc tam mirabilest? Plaut. Trin. 931. Quos locos adïsti? - Nimium mirimodis mirabiles. Terenz Heaut. tim. 387 Et vos esse istius modi et nos non esse haut mirabilest. Accius Didasc. 11 Falsidica audax gnati mater pessumi odibilis natura impos excors et fera. Liv. Andr. tr. 7 Iamne oculos specie laetavisti optabili. Plaut. Bacch. 159 Compendium edepol haud aetati optabile fecisti. Afran. tog. 166 Retinebitur viri hac voluntate unica probabili. Plaut. Aul. 633 Verberabilissume, etiam rogitas? (setzt wohl die Existenz des für das archaische Latein nicht sicher bezeugten - vergl. aber oben Accius tr. 613 - venerabilis voraus).

Bekanntlich kann im Verbum das Passiv reflexive oder intransitive Bedeutung annehmen: moveri "sich bewegen", volvi „rollen“. Derselbe Vorgang zeigt sich auch in Adjektiven auf bilis: Plaut. Mil. 629 Clare oculis video, sum pernix, manibus pedibus mobilis (,pernix pedibus, manibus mobilis" Bugge). Pacuv. tr. 367 Saxoque instare in globoso praedicant volubilei (welcher rollen kann).

Bei den form- und bedeutungsverwandten Adjektiven auf tilis schwindet bisweilen der Begriff der Möglichkeit, so daß nur reine Passivbedeutung übrig bleibt. Bei den Adjektiven auf bilis ist das nach meiner Ansicht im archaischen Latein, abgesehen von nobilis, nicht der Fall. Allerdings steht intestabilis an zwei oben citirten Plautusstellen (Curc. 30, Mil. 1414) im Wortspiel für intestatus ,entmannt" aber in seiner eigentlichen Bedeutung "nicht als Zeuge aufstellbar" ist es modal gefärbt. 
Vermuthen könnte man reine Passivbedeutung bei Plaut. Cist. 1, 1, 63 (sepelibilis) und Plaut. Stich. 395 (exoptabilis), doch läßt sich an beiden Stellen, wie ich oben durch Uebersetzung gezeigt habe, Modalbedeutung in die Adjektive hineinlegen. Höchst auffallend ist dagegen Plaut. Epid. 577 Scio quid erres: quia vestitum atque ornatum inmutabilem (,mutabilem" Pius) habet haec. Hier steht inmutabilis ,verändert" gleichbedeutend mit inmutatus; diese Stelle ist so seltsam, daß ich inmutatilem, eine scherzhafte Bildung nach Analogie von vestis plumatilis und vestis cumatilis, vermuthen möchte.

Es bleiben noch die Adjektive nobilis (gnobilis), ignobilis und cognobilis. Nach Festus (S. $174^{\mathrm{b}}$ ) wurde im archaischen Latein nobilis für notus gebraucht, und das scheint richtig zu sein. Es kann diese Bedeutung in folgenden Stellen angenommen werden: Liv. Andr. com. 4 Ornamento incedunt gnobiles ignobiles (citirt von Festus, wir kömnen die Richtigkeit der Erklärung nicht controlliren). Plaut. Pseud. 592 Quis hic est, qui oculis meis obviam ignobilis obicitur (citirt von Festus, hier kömnte allenfalls modale Färbung hineingelegt werden: „so daß er nicht erkennbar ist") Plaut. Pseud. 964 Peregrina facies videtur hominis atque ignobilis (citirt von Festus, der vorhergenannten Stelle gleichartig). Plaut. Pseud. 1112 Cum his mihi nec locus nec sermo unquam convenit, neque is nobilis fui (die Stelle ist kritisch unsicher, aber die reine Passivbedeutung in nobilis scheint unzweifelhaft). Plaut. Rud. 619 Vindicate, ne inpiorum potior sit pollentia qnam innocentum, qui se scelere fieri nolunt nobilis. Terenz Heaut. tim. 227 Meast potens procax magnifica sumptuosa nobilis (stadtbekannt). Dagegen glaube ich in der von Festus a. a. O. citirten Stelle Accius tr. 283 deutlich Modalbedeutung zu erkemnen: Ergo med Argos referam, nam hic sum gnobilis, ne cui cognoscar noto. Mit Rücksicht auf den sich anschließenden Satz "ne cui cognoscar noto " wird man in gnobilis nicht ein Synonym für notus sehen dürfen, sondern wird übersetzen müssen ,denn hier kann ich erkannt werden". Die Modalbedeutung ist unzweifelhaft im Adjektiv cognobilis bei Cato ed. Jordan S. 26, 10 Itaque ego cognobiliorem (verständlich) cognitionem esse arbitror. Daß nobilis in manchen Fällen rein passiv gebraucht wird, hängt wohl damit zusammen, daß es noch häufiger sowohl die Modal- als auch die Passivbedeutung abgeworfen und die intransitive Bedeutnng "vornehm" angenom- 
men hat. Die Belegstellen aus dem archaischen Latein will ich nur aufzählen, nicht ausschreiben: Plaut. Cist. 1, 2, 10. Trin. 831. Terenz Eun. 204. 952. Heaut. tim. 609. Adelph. 15. 502. L. Calpurnius Piso fr. 27 (Peter). Ignobilis ist entsprechend gebraucht: Enn. tr. 166. Terenz Phorm. 120, wahrscheinlich auch Liv. Andr. com. 4 und Pacuv. tr. 221 . . ques sunt is? - Ignoti, nescio ques ignobiles, als Schimpfwort Plaut. Amph. 440 Vapulabis, ni hinc abis, ignobilis: (Variante: ignorabilis).

\section{Adjectiva auf bilis, die sich der Aktiv- bedeutung nähern.}

a. Adjektiva a uf bilis mit der Bedeutung intransitiver Zustandsverba. - Anscheinend scharf und klar ausgeprägt ist der Unterschied von Aktiv und Passiv, und doch stößt man, sobald man ihn genauer auf seinen Werth prüfen will, auf erhebliche Schwierigkeiten. Man kann z. B. zweifeln, ob Passiva wie amari und timeri wirklich Passivbedeutung haben. Denn weder die Person, welche geliebt, noch die, welche gefürchtet wird, ist leidend, im Gegentheil, sie ist in gewisser Hinsicht aktiv, denn sie verursacht Liebe und Furcht. Noch schwieriger erscheint es, eine scharfe Grenze zwischen den beiden Genera zu ziehen, sobald man einen Unterschied innerhalb der Verba mit Aktivform beachtet, welcher dem von Aktiv und Passiv sehr nahe kommt. Denn viele Intransitiva verhalten sich zu bedeutungsverwandten Transitivis nahezu wie Passiva zu Aktivis, z. B. das intransitive cuere ,stürzen d. i. gestürzt werden" zum transitiven ruere ,stürzen d. i. stürzen machen". Bekannt ist ja, daß im Griechischen Intransitiva geradezu als Passiva verwendet werden können ( $9 \alpha v \varepsilon \check{\imath} v$ viró $\tau \iota v o \varsigma)$ und daß auch im Lateinischen Passiva zu Intransitivis werden können (effundi "fließen", aus dem Medium darf man das nicht erklären wollen). So erscheinen denn Intransitiva mit passiver Bedeutung und eigentliche Passiva als zwei Nuancen des Passivs gegenüber den aktive Bedeutung enthaltenden Transitivis. Ein Passiv von einem solchen Intransitivum sollte daher streng genommen nicht möglich sein, denn das Intransitivum ist schon passiv; wenn 
von ihm trotzdem ein impersonelles Passiv gebildet wird z. B. ventim est "man kam", so ist das eine Nachbildung nach datur "es wird gegeben d. h. man gibt" und dergleichen mehr, welche nur conventionell als Passiv gilt.

Ein dem Passiv nahe stehendes Intransitivum habe ich schon erwähnt nämlich volubilis, bei demselben war die intransitive Bedeutung aus der passiven entstanden. Es bleiben noch die Adjektive, welche von solchen Verben herzuleiten sind, die schon in der aktiven Form intransitiv sind. Die Scheidung von wirklicher und moralischer Möglichkeit braucht von nun an nicht mehr durchgeführt zu werden : so viel ich sehe, findet sich moralische Möglichkeit nur in den Adjektiven auf bilis mit echter Passivbedeutung. Die Beispiele für Adjektive auf bilis mit intransitiver Bedeutung sind: Accius tr. 264 Alternabilem divitiam partissent (alternabilem ist Conjektur Ribbecks für aeternabilem, das Verbum alternare ist erst seit Vergil belegt). Plaut. Capt. $402 \mathrm{Me} \mathrm{hic} \mathrm{pol} \mathrm{et} \mathrm{te} \mathrm{tute} \mathrm{audacter} \mathrm{dicito,} \mathrm{Tyndaze,} \mathrm{inter} \mathrm{nos} \mathrm{fuisse}$ ingenio haud discordabil. Plaut. Mil. 543 Nunc demum scio me fuisse excordem caecum incogitabilem. Terenz Hec. 284 Quanto fuerat praestabilius ubivis gentium agere aetatem quam huc redire. Plaut. Pers. 837 Hic faceret te prostibilem propediem (prostibilis wird gewöhnlich für ein Substantiv gehalten). Cato R. r. 35, 2 Qui locum novus erit aut qui restibilis fieri poterit. . . et qui locus restibilis crassitudine fieri potest. Plaut. Aul. 233 Neutrubi habeam stabile stabulum, si quid divorti fuat. Plaut. Bacch. 520 Profecto stabilest me patri aurum reddere. Plant. Merc. 653 Quae patria aut domus tibi stabilis esse poterit? Cato R. r. prooem. 4 Quaestus stabilissimus. Terenz Adelph. 66 Et errat longe mea quidem sententia, qui imperium credat gravius esse aut stabilius, vi quod fit.

b. Adjektiva a f bilis mit instrumentaler Bede utung. - Liest man bei Terenz Phorm. 226 causa vincibilis, so pflegt man in das Adjektiv Aktivbedeutung zu legen und also zu übersetzen ,eine Sache, die siegen kann". Ich halte das für unrichtig oder wenigstens ungenau. Ein Abstraktum wie causa ist an und für sich einer aktiven Thätigkeit garnicht fähig; wir pflegen zwar in unserer Muttersprache durch eine Redeweise, die wir als bildlich kaum noch empfinden, fast ohne Beschränkung sächlichen und abstrakten Dingen aktive Handlungen zuzuschreiben, aber der Römer der archaischen 
Zeit dachte darin strenger. Da nun ferner die Adjektiva auf bilis überwiegend passiv sind, so würde ich übersetzen ,eine Sache, mit welcher man siegen kann", indem ich annehme, daß wir hier eine besondere Art der Passivität, oder wenn man will der Aktivität, haben, welche ich die instrumentale nennen will. Es ist dies diejenige Art der Passivität, welche wir erhalten, wenn wir einen Akkusativ des inneren Objekts zum Subjekt des Passivs machen: der Ausdruck vincere causam ${ }^{3}$ )

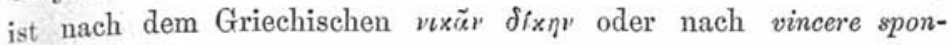
sionem bei Cicero pro Caec. 31,91 und 32,92 denkbar, das Passiv dazu wäre causa vincitur, und dem entspricht causa vincibilis. Der Akkusativ des inneren Objekts bei transitiven Verben ist nämlich nach meiner Ansicht immer instrumental und steht daher auf der Grenze von Aktiv und Passiv. Als Akkusativ des inneren Objekts betrachte ich auch den sächlichen Akkusativ bei Verben wie docere: haben wir den Satz grammaticus docet puerum litteras, so ist grammaticus aktiv und puerum passiv, litteras aber ist weder aktiv noch passiv sondern instrumental, denn passiv ist der Akkusativ litteras gewiß nicht, die litterae werden nicht unterrichtet, sie sind vielmehr in gewisser Hinsicht aktiv, sie unterrichten den Knaben gemeinschaftlich mit dem Lehrer, aber freilich nicht selbstthätig sondern als Werkzeug in der Hand des Lehrers. Diese instrumentale Passivität ist in den Adjektiven auf bilis nicht selten und keineswegs auf die Fälle beschränkt, wo das Stammverbum einen Akkusativ des inneren Objekts zu sich nehmen kann: Plaut. Mil. 1139 Date operam adiutabilem (opera adiutabilis heißt nicht opera quae adiutet sondern opera qua adiutetis, denn die Hülfe kann nur helfen als Mittel in der Hand einer thätigen Person). Plaut. Pers. 673 Edepol dedisti, virgo, operam adiutabilem (adiutabilem ist sichere Emendation). Accius tr. 551 Flebilis voces refert. Plaut. Epid. 342 Pro di inmortales, mi hunc diem ut dedistis luculentum, ut facilem atque inpetrabilem! (die instrumentale Passivität ist hier unzweifelhaft, natïrlich meint Plautus nicht, daB der Tag etwas erreichen kann, das wäre ganz unlateinisch gedacht, sondern daß man an dem Tage d. i. durch den Tag etwas erreichen kann). Plaut. Pers. $712 \mathrm{Ne} \mathrm{hic}$

3) Georges citirt noch vincere causam aus Ovid, aber dort ist es interpolirt. 
tibi dies inluxit luerificabilis (,durch welchen du dir Gewinn erwerben kannst", könnte aber auch ,gewinnbringend" heißen und also zur folgenden Classe gehören). 'Plaut. Cas. 4, 1, 3 Hic intus funt ludi ludificabiles (durch welche man foppen kann). Plaut. Asin. 792 Neque ullum verbum faciat perplexabile (durch welches er Verwirrung anrichten kann). Terenz Phorm. 961 Nunc quod ipsa ex aliis auditura sit, Chremes, id nosmet indicare placabilius est. Terenz Adelph. 608 Quapropter te ipsum purgare ipsi coram placabilius est. Terenz Phorm. 226 Iustam illam causam facilem vincibilem optumam.

In den genannten Beispielen spricht schon der Umstand, daß die Adjektiva mit Sachsubstantiven, die eigentlich gar nicht aktiv gedacht werden können, verbunden sind, dagegen, aktive Bedeutung in ihnen anzunehmen. Ausgelassen aber habe ich zwei Stellen von anderer Art, nämlich Plaut. Merc. 605 Inpetrabilior qui virat, nullus est. Plaut. Most. 1162 Non potuit venire oratar magis ad me inpetrabilis. Da sich, wie sich ergeben wird, wirklich aktive Bedeutung in den Adjektiven auf bilis sonst nicht findet, so wird man auch hier das Adjektiv inpetrabilis nicht als aktiv sondern als instrumental auffassen müssen wie in der erwähnten Stelle Plaut. Epid. 342 dies inpetrabilis ,ein Tag, durch den man etwas erreichen kann". So wird man Merc. 605 übersetzen müssen ,es lebt niemand, durch den man leichter etwas erlangen kann", in der That sollte ja der Jüngling, von dem die Rede ist, nicht für sich selbst etwas erreichen sondern für den Redenden. Ebenso steht es mit Most. 1162: orator magis inpetrabilis ist zu übersetzen, ein Sprecher, durch den man leichter etwas erlangen kann", denn auch er will nicht für sich sondern für einen anderen etwas durchsetzen. Wir werden also auch in diesen beiden Beispielen, obwohl das Adjektiv mit einem persönlichen Substantiv, welches an sich aktiv gedacht sein könnte, verbunden ist, instrumentale Bedeutung annehmen müssen.

c. Adjektiva a uf bilis mit kausaler Bedeutung. - Ich glaubte die instrumentalen Adjektiva nicht als aktiv bezeichnen zu dürfen, weil die Substantiva, mit denen sie verbunden werden, nicht selbständig sondern nur als Werkzeuge in der Hand der handelnden Person thätig erscheinen. Es gibt aber noch eine andere Art der Aktivität, welche dem Passiv ebenso nahe steht und welche ich als die $\mathrm{kausale}$ bezeichnen 
will. Dieselbe erkenne ich z. B. im Adjektiv terribitis: ein Gegenstand, welcher Schrecken verursacht, braucht keineswegs aktiv thätig zu sein, er kann vielmehr nur als Werkzeug in der Hand des Geschicks oder des Zufalls dienen, während er selbst vollkommen unthätig d. h. passiv ist. Diese causale Bedeutung ist mit der instrumentalen nahe verwandt; bei einigen Beispielen, die ich für die instrumentale Aktivität gegeben habe, konnte man zweifeln, ob nicht vielmehr die kausale in ihnen zu erkennen sei, ob man z. B. ludi ludificabiles (Plaut. Cas. 4, 1, 3) iibersetzt durch "Spiele, durch welche jemand foppt" oder ,Spiele, durch welche gefoppt wird" (mit unbestimmtem logischem Subjekt, denn wirklich aktiv können die ludi niemals sein) kommt fast auf dasselbe hinaus: in beiden Fällen ist ludificabilis in gewisser Hinsicht zwar aktiv, aber die in ihm liegende Thätigkeit kann verglichen werden mit der Thätigkeit einer Maschine oder eines Automaten, der nur scheinbar handelnd auftritt. Suchte ich vorhin die Verwandtschaft der instrumentalen Bedeutung mit der passiven dadurch zu beweisen, daß ich auf ihre Corresponsion mit dem inneren Objekt hinwies, so läßt sich bei der kausalen in analoger Weise der Beweis führen. Es werden manche Verba mit einem Akkusativ construirt (und zwar mit einem Akkusativ, der meist als Akkusativ des äuBeren Objekts gilt), obwohl sie eigentlich einen Zustand und keine Thätigkeit bezeichnen, dahin gehören z. B. amare ("lieben" ist ein Zustand, das Transitivum dazu wäre ,lieben machen"), timere (,in Furcht sein", als Transitivum dazu kann man, wenn man von dem Unterschied von Furcht und Schrecken absieht, terrere betrachten), fugere (das Transitivum dazu ist fugare), uti (uti aliquid: Nutzen haben von etwas), horrere (horrere aliquid: schaudern vor etwas), gaudere (gaudere aliquid) u. a. m. Bei manchen dieser Verba weist schon die Form (timere gaudere horrere, vergl. albere florere vigere) darauf hin, daß sie ursprünglich intransitiv waren. Der Akkusativ, der bei diesen Verben steht, ist eigentlich transitiv, zwar nicht aktiv, aber doch kausal, allerdings sind viele ganz in die Analogie der transitiv-aktiven Verba übergeführt, so amare timere fugere, nur zögernd folgten uti (wo die Construction mit dem Akkusativ zurückgedrängt wurde), horrere (von welchem man horrendus ableitete) gaudere (erst spät bildete man gaudendus). Man kann also sagen, die 
kausale Bedeutung entspricht derjenigen Passivität, welche man erhielte, wenn man das kausale Objekt von Verben wie uti horrere, gaudere zum Subjekt des Passivs machen würde. Besonders interessant ist utibilis; dasselbe enthält deutlich transitive, d. h. kausale Bedeutung: es heißt „das, was nützen kann", nicht "das was benützt werden kann" und correspondirt mit dem kausalen Objekt bei uti, z. B. kann quid minus utibile fuit (Terenz Phorm. 695) umschrieben werden durch quid minus uti oportuit. Ebenso steht es mit horribilis, auch es correspondirt. mit dem causalen Objekt bei horrere, während in den übrigen Beispielen die Adjectiva mit dem causalen Subjekt correspondiren z. B. terribilis. Die Adjektiva mit kausaler Bedeutung sind: Accius tr. 158 Sed pervico Aiax animo atque advorsabili (advorsabili ist. unsicher). Plaut. Bacch. 52 Non ego istuc facinus mihi, mulier, conducibile esse arbitror. Plaut. Cist. 1, 1, 79 Matronae magis conducibile est istuc, mea Silenium. Plaut. Epid. 256 Reperiamus aliquid calidi conducihilis consili. Plaut. Epid. 260 Dederim vobis consilium catum . . atque ad eam rem conducibile. Plaut Epid. 388 Fuit conducibile hoc quidem mea sententia. Plaut. Trin. 25 Amicum castigare ob meritam noxiam inmoenest facinus, verum in aetate utile et conducibile. Plaut. Trin. 36 Ita vincunt illud conducibile gratiae. Plaut. Epid. 606 Si invenio, exitiabilem ego illi faciam hunc ut fiat diem (exitiabilem ego faciam ut hic fiat dies hat der Ambrosianus, darnach hat Geppert nicht ohne Wahrscheinlichkeit vermuthet: exitialis illi ego etc.). Lucilius 153 (Bährens) Flebile cepe simul lacrimosaeque ordine tallae (die Bedeutung von flebilis ist hier eine andere als oben in flebiles voces bei Accius tr. 551 ; hier ist das Adjektiv kausal und entspricht also einem kausalen Objekt wie quid fles "warum weinst du?", dort war es instrumental und entsprach einem inneren Objekt wie flere carmina bei Columella). Lucilius 1 Aetheris et terrae genitabile quaerere tempus (dies Beispiel ist sehr significant: activ, "gebärend", ist genitabile tempus natürlich nicht, aber ebenso wenig echtes Passiv). Accius tr. 800 vim torvam aspecti, atque horribilem (welche schaudern machen kann). Accius tr. 617 Nam etsi opertus squalitate est luctuque horrificabili (horriflcali ist überliefert). Plaut. Cist. 2, 2, 3 Ut illaec hodie quot modis sibi moderatrix fuit atque inmemorabilis (vergl. die vorstehende Stelle). Lucilius 481 Nunc ignobilitas his miserum, 
mirum ac mortificabile (man pflegt mortificabile in monstrificabile zu ändern). Ennius tr. 22 Mater terribilem minatur vitae cruciatum et necem. Ennius Ann. 95 At tuba terribili sonitu taratantara dixit. Satur. 462 Africa terribili tremit horrida terra tumultu (Variante: terribilis). Trag. inc. 96 Quae tam terribilis tua pectora turbat. Accius tr. 421 Pernici orbificor liberorum leto et tabificabili. Plaut. Epid. 21 Voluptabilem mihi nuntium tuo adventu adportas. Plaut. Bacch. 1 Quibus ingenium in animo utibilest. Plaut. Merc. 1005 Eamus intro: non utibilist hic locus (utibilest hic loqui Götz in der Anm.). Plaut. Mil. 612 Immo magis esse ad rem utibile non potest. Plaut. Most. 859 Servi, qui quom culpa carent, tamen malum metuont, hi solent esse utibiles eris. Plaut. Trin. 748 Vide si hoc utibile magis atque in rem deputas. Terenz Phorm. 690 Quid minus utibile fuit quam hoc ulcus tangere.

Nichts entnehmen für die Bedeutung läßt sich aus folgenden Fragmenten, die Adjektiva auf bilis aus dem Zusammenhang gerissen enthalten: Pacuv. Antiop. fr. XIV cor luctificabile. Cato ed. Jordan S. 109, 12 mobiliorem, nobiliorem. Festus führt medibilis an.

Ebenso wenig nützen uns die Adverbia auf biliter: Plaut. Pseud. 950 cruciabiliter (unsicher). Plaut. Mil. 260 dissimulabiliter. Naev. com. 35 exanimabiliter. Pacuv. tr. 15 . Accius praet. 11 minitabiliter. Accius tr. 258 indecorabiliter. Cato ed. Jordan S. 89, 6 imperabiliter. Plaut. Most. 24 pollucibiliter.

Ebensowenig die Substantiva auf bilitas: Plaut. Stich. 741 amabilitas. Plaut. Poen. 1174 amabititati. Plaut. Cist. 2, 1, 3 cruciabilitatibus. Lucil. 481 ignobilitas. Plaut. Rud. 933. Accius tr. 621. 643 nobilitas. Plaut. Capt. 299. Mil. 1319. Terenz Hec. 797 nobilitatem. Turpil. com. 208. Lucil. 198 nobilitate.

Ebenso wenig schließlich die abgeleiteten Verba: Plaut. Capt. 453 constabilivi. Terenz Ad. 771 constabilisses. Caecil. Stat. com. 192 mobilitat. Terenz Eun. 1021 nobilitas. Pacuv. tr. 120. Accius tr. 622 nobilitat. Titin. tog. 69 nobilitarent. C. I. L. I 38 nobilitavit. Pacuv. tr. 324 restibiliet. Ennius Ann. 28, 9 stabilibat. Accius praet. 40 stabiliverat. Ennius Ann. 55, 21 stabilita und das daraus abgeleitete Substantiv Accius tr. 210 stabilimen. 
Das Resultat, das wir gewonnen haben, ist also: Die Adjektiva auf bilis sind im archaischen Latein niemals wirklich aktiv, d. h. sie treten nie zu einem.Substantiv, das selbständig thätig gedacht wird, hinzu, niemals wird gesagt homo adiutabilis ,ein Mann, der helfen kann" 4) oder dergleichen, sie sind vielmehr entweder passiv oder sie enthalten gewisse Mittelstufen zwischen echtem Passiv und e chtem Aktiv, welche ich als die intransitive, instrumentale und causale Bedeutung bezeichnet habe. Mir scheint, daB wir dadurch einen interessanten Einblick in die Denkgesetze der lateinischen Sprache gethan haben: wir haben eine wesentlich andere Scheidung von Aktiv und Passiv getroffen, als im Verbum üblich ist, und zwar eine Scheidung, welche ich für eigentlich besser begründet und auch für älter halte. Für älter halte ich sie deshalb, weil sie besser in Einklang steht mit dem Unterschied, der zwischen Subjekt und Objekt besteht, und auf diesen Punkt will ich zum Schluß mit wenig Worten eingehen.

Es kann keinem Zweifel unterliegen, daß das Objekt eigentlich ein zweites Subjekt ist. Wie es geschehen konnte, daß von zwei zu einem gemeinschaftlichen Prädikat gehörigen Subjekten das eine zum Objekt herabgedrückt wurde, hat Paul, Principien der Sprachgeschichte, zweite Aufl., Halle 1886 S. 113 überzeugend dargelegt: „Wir können uns diesen ProceB am besten verdeutlichen an einem Satze wie ich rieche den Braten. Ohne persönliches Subjekt können wir auch noch sagen der Braten riecht. Wir können uns danach leicht in eine Zeit zurückversetzen, in welcher bei völligem Mangel jeglichen Casussuffixes und jeglicher Fixierung der Wortstellung in einem Satze wie ich riechen Braten oder Braten riechen ich die Wörter ich und Braten unter dieselbe allgemeine Kategorie des psychologischen Subjekts fielen. Die Verwandtschaft zwischen Subjekt und Objekt erhellt ja auch daraus, daß das letztere durch Umsetzung des Verbums in das Passivum zum ersteren gemacht werden kann". Ich will versuchen auf dieser Grundlage das Verhältniß von Subjekt zu Objekt etwas genauer zu prüfen ${ }^{5}$ ).

4) Das könnte höchstens heißen ,ein Mann, durch den geholfen werden kann", vergl. orator inpetrabilis.

5) Ich hoffe, daß es mir gelingen wird, zu zeigen, daß wir uns 
Für werthlos halte ich dabei das von Paul am Schluß der citirten Stelle hervorgehobene Kriterium, daß das Objekt bei Umsetzung des Verbums ins Passiv als Subjekt erscheint. Denn es gibt bekanntlich Objektsakkusative die nicht zum Subjekt des Passivs erhoben werden können ${ }^{6}$ ). Auch ist das Passiv, welches sich, darüber sind wohl alle einig, erst aus dem reflexiven Medium entwickelt hat, eine verhältnißmäßig junge Bildung. Vom Passiv müssen wir also zunächst ganz absehen, und einen der Entstehung des Passivs voraufliegenden Sprachzustand ins

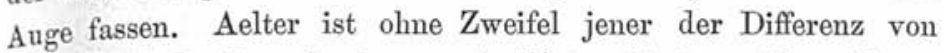
Aktiv und Passiv nahe kommende Unterschied von Transitivum und Intransitivum und dieser ist das wichtigste Kriterium, um die verschiedenen Arten des Objektsakkusativ zu sondern. Es ergeben sich vier Klassen:

bei der nichtssagenden Definition des Akkusativs, welche in der modernen Grammatik üblich geworden ist und welche z. B. bei Schmalz (ich bin weit entfernt, diesen verdienstvollen Mann dafür verantwortlich zu machen) in Iwan Müllers Handbuch II S. 262 folgenden Ausdruck findet ,, der Akkusativ ist ein rein verbaler Kasus und gibt lediglich die Modifikation des Prädikats" nicht zu beruhigen brauchen.

6) Dazu rechne ich nicht den lokalen Akkusativ des Zieles (Romam eo), derselbe ist vielmehr gar kein Objektsakkusativ mehr, sondern gehört in eine Kategorie mit den übrigen Casus obliqui, mit den prāpositionalen Wendungen und den Adverbien. Alle diese Elemente sind zwar aus dem Objekt hervorgegangen, aber thatsächlich geben sie nicht mehr eine objektive sondern eine attributive Bestimmung des Verbums. Diese attributive Funktion wird dadurch deutlich, da $B$ dieselben Elemente (die lateinische Sprache ist freilich mit dieser Uebertragung viel zurückhaltender als das Deutsche und Griechische) auch beim Nomen attributiv verwendet werden können: Plaut. Merc 257 navem ex Rhodo. Plaut. Pers. 385 nune hominum, ganz gewöhnlich der Ablativ qualitatis, auch der Genitiv ist wohl ursprünglich ein adverbialer Casus und nicht ein erstarrtes Adjektiv. Auch der lokale Akkusativ kann attributiv verwendet werden: domum reditus, Romam adventus und dergl., vergl. Dräger Syntax ${ }^{2}$ I $\$ 177$. Ganz anders steht es mit Redewendungen wie Quid tibi nos tactio est?, die im archaischen Latein gewöhnlich sind, vergl. Dräger a. a. O. $\S 163$. Bei denselben gehört der Objektsakkusativ nicht attributiv zum Verbalsubstantiv, sondern das Verbalsubstantiv wird mit der Copula zusammengefaßt, und dies zusammengesetzte Prădikat regiert den Akkusativ. Zu vergleichen sind hiermit Ausdrücke wie infitias ire aliquid: hier ist infitias prädikativ gebraucht und bildet mit ire zusammen einen neuen transitiven Verbalbegriff. Uebrigens glaube ich, daß Schmalz in Iwan Müllers Handbuch II S. 263 im Irrthum ist, wenn er diesen Akkusativ infitias als lokalen Akkusativ auffaßt und mit dem Akkusativ von Städte- und Ländernamen zusammenstellt: es ist ohne Zweifel ein Akkusativ des inneren Objekts und also zusammenzustellen mit ire viam. 
a. Transitives Subjekt und intransitives Objekt ${ }^{7}$ ). Hierher gehört die Mehrzahl der Verba, die ein direktes Objekt bei sich haben. Beispiele sind: Volvo lapides ich rolle, d. h. mache rollen, und daher rollen die Steine. Ago capellas ich treibe, und daher gehen die Ziegen. Mitto servum ich schicke, d. h. mache gehen, und daher geht der Sklave. Fugo hostes ich schlage in die Flucht, und daher fliehen die Feinde. Colo agrum ich pflege, und daher empfängt der Acker Pflege. Tego domum ich decke, und daher empfängt das Haus ein Dach.

b. Intransitives Subjekt und transitives Objekt: Maneo te ich bleibe, weil du bleiben machst. Gemo ve. strum malum ich seufze, weil euer Unglück seufzen macht. Gaudeo omnia haec ich freue mich, weil alles dies Freude veranlaßt. Tremo Iunonem ich zittere, weil Iuno zittern macht. Timeo mortem ich fürchte mich, weil der Tod fürchten macht. Patior vulnera ich leide, weil die Wunden leiden machen. Fugio hostem ich fliehe, weil der Feind fliehen macht.

c. Transitives Subjekt und transitives Objekt: Doceo artem ich lehre mit einer Kunst (dagegen mit intransitivem Objekt: doceo puerum). Rogo aliquid ich befrage mit etwas (dagegen mit intransitivem Objekt: rogo te). Sero hordeum ich säe mit Gerste (dagegen mit intransitivem Objekt: sero agrum) Vinco bellum ich siege nit einem Kriege (dagegen mit intransitivem Objekt: vinco hostem).

d. Intransitives Subjekt und intransitives Objekt: Somnio somnum ich träume mit einem Traum. Eo viam ich gehe mit einem Wege. Tremo artus ${ }^{8}$ ) ich zittere mit den Gliedern. Vivo vitam ich lebe mit dem Leben. Luceo facem ich leuchte mit einer Fackel. Stillo rorem ich tröpfele mit Than ${ }^{9}$ ).

7) Nehmen wir z. B. verto, welches transitiv und intransitiv sein kann (als noch kein Passiv existirte, konnte wahrscheinlich jedes Verbum nach Belieben transitiv oder intransitiv gebraucht werden, die Verbalwurzel wenigstens enthielt ohne Zweifel beide Bedeutungen), so ist leicht verstăndlich, deß zwei Sätze wie agricola vertit ,,der Landmann macht wenden" und bos vertit ,, der Ochse wendet sich" zusammengezogen werden konnten in agricola bovem vertit, ,der Landmann wendet den Ochsen", wobei das Verbum im Verhăltniß zu agricola transitiv, im Verhältniß zn bovem intransitiv ist.

8) Das ist ein Graecismus, aber dieser Umstand thut nichts zur Sache, denn im Griechischen steht es nicht anders.

9) Die Klassen $a$ und $b$ enthalten die äußeren, die Klassen $c$ und 
Es ist selbstverständlich, daß dasjenige, was vom Objekt ausgesagt ist, stets in causaler Beziehung steht zu demjenigen, was vom Subjekt ausgesagt ist. Ich habe das auch durch die Umschreibung anzudeuten gesucht und habe z. B. volvo lapides paraphrasirt durch, ,ich rolle und daher rollen die Steine". Bemerkenswerth ist aber, daß wir in den vier Klassen, die ich unterschieden habe, die vier Species des Kausalitätsverhältnisses, Zoeck, Ursache, Mittel, Art und Weise $\left.{ }^{10}\right)$, finden, und ich schlage daher folgende Bezeichnungsweisen vor:

Finaler Akkusativ (Zweck): volvo lapides (Klasse a).

Kausaler Akkusativ (Grund): fugio hostem (Klasse b).

Instrumentaler Akkusativ (Mittel): doceo artem (Klasse c).

Modaler Akkusativ (Art und Weise): stillo rorem (Klasse d).

In dieses System wurde Verwirrung gebracht durch die Ausbildung des Passivs. Das Verhältniß des Aktivs zum Passiv entspricht nur dem des Subjekts zum finalen Objekt. Die übrigen Arten des Objekts wurden entweder gewaltsam, den Gesetzen strengen Denkens zuwider, umgedeutet, so bei timere, amare etc. vergl. oben S. 283, auch bei docere, rogare u. a. m., oder sie wurden nicht oder doch nicht deutlich mehr als Objekte empfunden.

Wir haben bisher einen wesentlichen Unterschied zwischen Subjekt und Objekt nicht gefunden: der Gegensatz von Aktiv und Passiv ist erst nachträglich und unvollkommen hineingetragen worden, der Gegensatz von Transitivum und Intransitivum ist zwar wichtig für die Scheidung der verschiedenen Klassen aber das Subjekt kann so gut wie das Objekt transitiv oder intransitiv sein. Das unterscheidende Merkmal ist vielmehr: der Bewußte, Thätige wird als Subjekt, das Unempfindliche, nur Leidende oder nur mechanisch Thätige als Objekt bevorzugt ${ }^{11}$ ).

Ich kehre zu dem Ausgangspunkt dieser Untersuchung zurück: Die Bedeutung der Adjektiva auf bilis steht in naher Beziehung zur grammatischen Kategorie des Objekts. Wie das

$d$ die inneren Objekte. Stillo rorem und dergl. betrachte ich unbedenklich als Akkusative des inneren Objekts, vergl. Delbrück, Synt. Forsch. 4 S. 30 unten.

10) Vergl. Wundt Logik I S. 132 und 182.

11) Ganz consequent ist das nicht durchgeführt, es widersprechen z. B. die Impersonalia piget, pudet etc.

Philologus. N. F. Bd. I, 2. 
Objekt entweder leidend (Klasse $a$ und $d$ ) oder nur mechanisch thätig ist (Klasse $b$ und $c$ ), so schließen sich die Adjektiva auf bilis nicht an bewußt und selbstständig thätig sondern an leidend oder nur mechanisch thätig gedachte Substantiva an; besonders wichtig ist die Beziehung der instrumentalen und kausalen Adjektiva zu den instrumentalen und kausalen Akkusasiven. Wir sehen also, daß wir uns bei den Adjektiven auf bilis ebenso wie bei der Lehre vom Akkusativ von den ïblichen Anschauungen über die genera verbi freizumachen haben ${ }^{12}$ ).

12) Die griechischen Adjectiva auf -tos, die sich in einem großen Theil ihres Bedcutungsumfanges mit den lateinischen auf -bilis decken, werden demnächst von Charles Edward Bishop aus Petersburg in Nord-Amerika behandelt werden und es wird sich dabei eine Bestätigung meiner Resultate ergeben.

Leipzig.

Friedrich Hanssen.

\section{Zu Apuleius.}

Apol. cap. II p. 4, 6 (Krueg.). igitur Pontianum fratris sui filium, quem paulo prius occisum a me clamitarat, postquam ad subscribendum compellitur, ilico oblitus est. [de morte cognati adolescentis] sin subito taceret tanti criminis descriptionem, tamen ne omnino desistere videretur calumnia magiae, quae facilius infamatur quam probatur, eam solum sibi delegit ad accusandum. Die Stelle läßt sich auf eine weniger gewaltsame Weise heilen, indem man subito tacerem zu subit tacere in ändert und mit $\mathrm{V}$ descriptione liest. Demnach lautet sie oblitus est . de morte cognati adolescentis subiit tacere in tanti criminis descriptione: tamen, ne omnino desistere videretur, calumniam magiae, quae facilius infamatur quam probatur, eam solum sibi delegit ad accusandum. Zur. Wiederaufnahme des Objekts durch das Determinativum nach einem Relativsatze vergleiche man IIII pag. 7, 6 Pythagoram, qui primum se esse philosophum nuncuparit, eum sui saeculi excellentissima forma fuisse; L pag. 60, 22 quod animi partem rationalem, quae longe sanctissima est, eam violet.

Graz.

M. Petschenig. 